\title{
Numerical Simulations of QPOs in Bondi-Hoyle type accretion: the case of SgrA*
}

\section{O. Donmez}

Nigde University, Department of Physics, Nigde Turkey, 51200

E-mail: edonmez@nigde.edu.tr

\section{O. Zanotti}

Max-Planck-Institut für Gravitationsphysik, Albert Einstein Institut, Golm, Germany

E-mail: zanotti@aei.mpg.de

\section{Rezzolla}

Max-Planck-Institut für Gravitationsphysik, Albert Einstein Institut, Golm, Germany

Department of Physics, Louisiana State University, Baton Rouge, LA USA

E-mail: rezzolladaei.mpg.de

\begin{abstract}
We show the results of two-dimensional and general-relativistic simulations of Bondi-Hoyle accretion flows onto a rotating black hole, revealing the existence of quasi-periodic oscillations (QPOs) in the shock cone that develops in the downstream part of the flow. The frequencies of these modes depend on the black-hole spin and on the properties of the flow, and scale linearly with the inverse of the black-hole mass. Our results may be relevant for explaining the detection of QPOs in Sagittarius A*, once such detection is confirmed by further observations. We also report the first numerical evidence for the occurrence of the flip-flop instability, which can affect the shock cone, in a relativistic Bondi-Hoyle.
\end{abstract}

25th Texas Symposium on Relativistic Astrophysics - TEXAS 2010

December 06-10, 2010

Heidelberg, Germany 


\section{Contents}

1. Introduction 2

2. Numerical approach and initial conditions 2

3. Quasi Periodic Oscillations 3

4. Discussion and Conclusions

\section{Introduction}

Non-spherical accretion is astrophysically relevant when strong winds accrete onto a compact object with very small angular momentum. This is for instance the case of High Mass X-ray binaries and of young stellar systems orbiting in the gravitational potential of their birth cluster. Hoyle \& Littleton and Bondi \& Hoyle [1, 2] were the first to consider the accretion properties of a black hole when it moves relative to a uniform gas cloud. A key feature of the Bondi-Hoyle flow, which has been the subject of several numerical investigations, starting from [ [3] and followed by [4, 5, 6], is the stability against tangential velocities of the shock cone that forms in the downstream region of the flow. Such instability, called flip-flop instability, manifests in the oscillation of the shock cone and has been studied extensively in the Newtonian regime. In the relativistic regime, the first two-dimensional simulations of Bondi-Hoyle accretion were performed by [7] and subsequently by [8, 9, 10, 11], who, however, do not report any occurrence of the flip flop instability.

An additional and very relevant physical effect related to the Bondi-Hoyle flow is represented by the possibility that the shock cone may provide a natural cavity for the development and confinement of oscillation modes of sonic nature, namely Quasi Periodic Oscillations (QPOs). We perform two dimensional general relativistic hydrodynamics simulations by focusing on the dynamical behavior of the Bondi-Hoyle shock cone under a wide range of parameters. The QPOs that we have found have typical frequencies in the range from $10^{-5} \mathrm{~Hz}$ to $10^{-3} \mathrm{~Hz}$ for a black hole with $M=10^{6} M_{\odot}$ (and in the range from $1 \mathrm{~Hz}$ to $10^{2} \mathrm{~Hz}$ for a black hole with $M=10 M_{\odot}$ ). As a result, they can become relevant for the interpretation of QPOs observed both in the galactic center and in high-mass X-ray binaries.

\section{Numerical approach and initial conditions}

We have solved the equations of general relativistic hydrodynamics with a code described in full details in [12]. The code is based on the conservative formulation of the equations and adopts standard high resolution shock-capturing methods by resorting to Riemann solvers. A minmod linear algorithm for the reconstruction of the left and right states at each interface between adjacent 


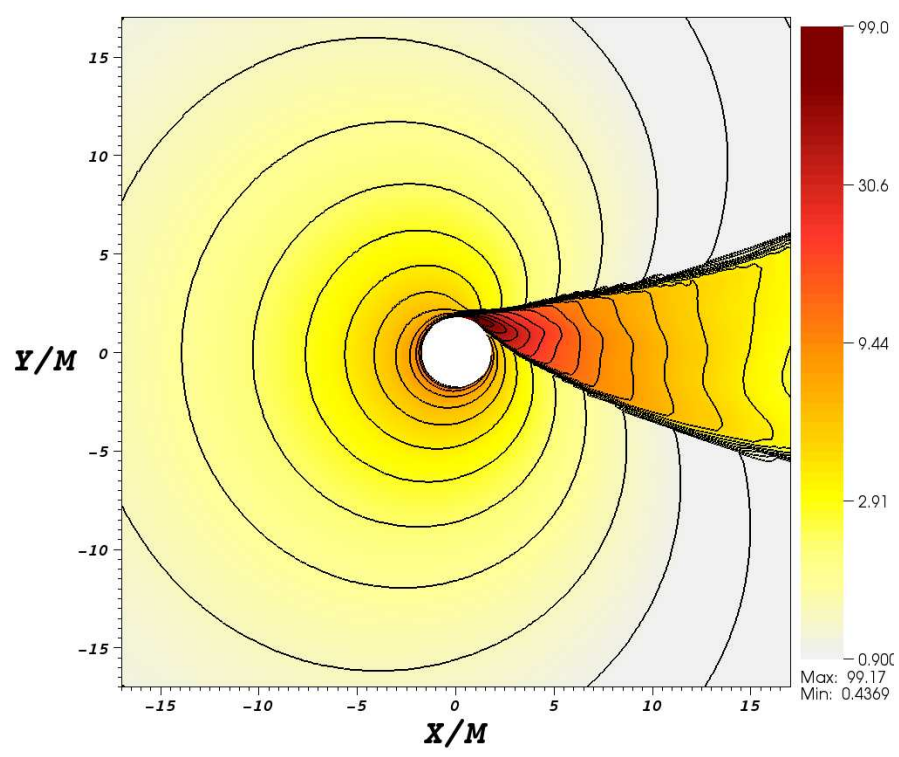

Figure 1: Rest mass density distribution, showing the typical shock cone in the downstream part of the flow, in a model with velocity $V_{\infty}=0.5$ and black hole spin $a / M=0.9$.

numerical cells is adopted, while the numerical fluxes are computed with the Marquina flux formula. The initial conditions are those typical of Bondi-Hoyle flow, hence with a velocity field that, in Boyer-Lindquist coordinates, can be written in terms of an asymptotic velocity $V_{\infty}$ as

$$
\begin{aligned}
V^{r} & =\sqrt{\gamma^{r r}} V_{\infty} \cos (\phi), \\
V^{\phi} & =-\sqrt{\gamma^{\phi \phi}} V_{\infty} \sin (\phi) .
\end{aligned}
$$

These relations guarantee that the velocity of the injected gas at infinity is parallel to the $x$-direction, while $V^{2} \equiv V_{i} V^{i}=V_{\infty}^{2}$ everywhere in the flow. During the evolution additional matter is injected supersonically from the outer boundary in the upstream region with the same analytic prescription of (2.1) and (2.2), thus creating a continuous wind at large distances. The initial density and pressure profiles are adjusted to make the sound speed equal to a required value, which we choose to be $c_{s, \infty}=0.1$. The simulations are performed on the equatorial plane, namely $\theta=\pi / 2$, using $N_{r} \times N_{\phi}$ uniformly spaced zones in the radial and angular directions, respectively, covering a computational domain extending from $r_{\text {min }}$ just beyond the horizon to $r_{\max }=43 M$ and from 0 to $2 \pi$. For our fiducial simulation we have chosen $N_{r}=512$ and $N_{\phi}=256$.

\section{Quasi Periodic Oscillations}

The typical flow pattern of the Bondi-Hoyle flow is reported in Fig. 1 for a model with $V_{\infty}=0.9$ and black hole spin $a / M=0.9$. The shape of the shock cone that forms in the downstream region of the flow depends sensitively on the spin of the black hole. If the black hole is not spinning, 


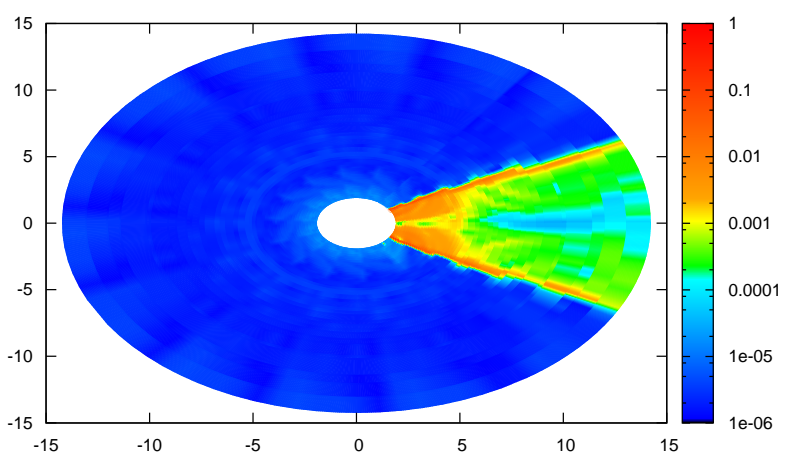

Figure 2: Power Spectral Density of the fundamental mode of oscillation in a model with $V_{\infty}=0.2$ and black hole spin $a / M=0.0$.

in fact, the shock cone is perfectly symmetric about the $\phi=0$ direction. On the other hand, if the black hole is spinning, the induced frame-dragging effect produces a "wrapping" of the shock cone, as clearly reported in Fig. 1. Once the physical system, as described above, has relaxed to the stationary state, Quasi Periodic Oscillations are produced in the shock cone. In order to extract as much information as possible about the emerged phenomenology and provide a first physical explanation, we have carried out an extensive Fourier analysis of the different dynamical quantities. We have found that several modes of oscillations are excited, essentially independent of the radial position. This is a clear indication that the modes are local waves but they are global eigenmodes of the system. Moreover, frequent nonlinear couplings among modes take place, so that if the system has eigenfrequencies $\omega_{i}$, the non-linearity of the equations will also produce modes at frequencies $\omega_{i} \pm \omega_{j}$, with amplitudes which are proportional to the product of the amplitudes of the combining frequencies. In particular, modes with frequencies in the integer ratios $2: 1$ and $3: 1$ are easily produced. As a representative example, Fig. 2 reports the Power Spectral Density of the fundamental model of oscillation detected for a model with $V_{\infty}=0.2$ and black hole spin $a / M=0$. The global nature of the oscillation modes inside the shock cone is clearly shown. Note that the intensity is computed over the whole computational domain, but it has a non-negligible amplitude only inside the shock cone, and this amplitude becomes increasingly stronger near the black hole. What shown in Fig. 目 is not specific to the mode shown and we have verified that the spatial distribution of the intensity manifests a similar pattern for all of the other modes.

\section{Discussion and Conclusions}

Two major results have been obtained through the research presented here. The first one consists in the first detection of Quasi Periodic Oscillations in the shock cone developing in the downstream region of a Bondi-Hoyle flow. These modes of oscillations are global in the sense that they represent harmonic oscillations across the cavity, but have amplitudes that are larger in the region very close to the black hole, namely for $r \lesssim 10 M$. While the black hole spin influences 
the absolute frequencies of the trapped modes, which lie in the range from $1 \mathrm{~Hz}$ to $500 \mathrm{~Hz}$ for a representative black hole of mass $M=10 M_{\odot}$, it does not affect the fact that they appear in a series of integer numbers $1: 2: 3$. This is because nonlinear coupling of modes is common in systems governed by nonlinear equations, and once a mode is excited, all of its integer multiples are also excited, thus producing a wide range of possible ratios of integer numbers. This new feature that we have put into evidence could be at the origin of the phenomenology reported in $\mathrm{Sgr} \mathrm{A}^{*}$, where both QPOs [14] and Bondi-Hoyle accretion [16] are likely to be present. Remarkably, a large family of linear combinations of modes has been identified recently by Aschenbach [15] after analyzing data about a high-level X-ray activity of Sgr A* observed with XMM-Newton. More robust observations will possibly confirm our interpretation.

The second result that we have obtained is the evidence for the occurrence of the so called flip-flop instability in a general relativistic context. More specifically, for a fixed choice of the black hole spin and of the sound speed, there exists a critical value of the asymptotic flow velocity at which the shock cone undergoes large-scale and coherent oscillations. When this happens, the opening angle of the shock cone reaches its maximum value, the accretion rate increases considerably and is no longer stationary [17].

\section{Acknowledgments}

The numerical calculations were performed at the National Center for High Performance Computing of Turkey (UYBHM) under grant number 10022007 and TUBITAK ULAKBIM, HighPerformance and Grid-Computing Center (TR-Grid e-Infrastructure). This work was supported in part by the DFG grant SFB/Transregio 7 and TUBITAK-BIDEP, 2219.

\section{References}

[1] F. Hoyle, R. A. Lyttleton, The effect of interstellar matter on climatic variation, 1939, in proceedings of the Cambridge philosophical society Vol. 35. pp 405

[2] H. Bondi, F. Hoyle, On the mechanism of accretion by stars, 1944, Mon. Not. R. Astron. Soc., 104, 273

[3] T. Matsuda, M. Inoue, K. Sawada, Spin-up and spin-down of an accreting compact object, 1987, Mon. Not. Roy. Astr. Soc., 226, 785

[4] B. A. Fryxell, R. E. Taam, Numerical simulation of nonaxisymmetric adiabatic accretion flow, 1988, Astrophys. J., 335, 862

[5] K. Sawada, T. Matsuda, U. Anzer, G. Boerner, M. Livio, Inhomogeneous wind accretion Comparison between $3 D$ and 2D computations, 1989, Astron. and Astrophys., 221, 263

[6] J. S. Benensohn, D. Q. Lamb, R. E. Taam, Hydrodynamical Studies of Wind Accretion onto Compact Objects: Two-dimensional Calculations, 1997, Astrop. J., 478, 723

[7] L. I. Petrich, S. L. Shapiro, R. F. Stark, S. A. Teukolsky, Accretion onto a moving black hole - A fully relativistic treatment, 1989, Astrophys. J., 336, 313

[8] J. A. Font, J. M. Ibáñez, A Numerical Study of Relativistic Bondi-Hoyle Accretion onto a Moving Black Hole: Axisymmetric Computations in a Schwarzschild Background, 1998b, Astrophys. J., 494, 297 
[9] J. A. Font, J. M. . Ibáñez, Papadopoulos P., A “Horizon-adapted” Approach to the Study of Relativistic Accretion Flows onto Rotating Black Holes, 1998, Astrophys. J, 507, L67

[10] J. A. Font, J. M. Ibáñez, Papadopoulos P., Non-axisymmetric relativistic Bondi-Hoyle accretion on to a Kerr black hole, 1999, Mon. Not. R. Astron. Soc., 305, 920

[11] B. D. Farris, Y. T. Liu, S. L. Shapiro, Binary black hole mergers in gaseous environments: "Binary Bondi" and "binary Bondi-Hoyle-Lyttleton" accretion, 2010, Phys. Rev. D, 81, 084008

[12] O. Dönmez, 2004, Astrophys. Spac. Sci., Code Development of Three-Dimensional General Relativistic Hydrodynamics with AMR (Adaptive-Mesh Refinement) and Results from Special and General Relativistic Hydrodynamics, 293, 323

[13] J. A. Font, J. M. Ibáñez, Non-axisymmetric relativistic Bondi-Hoyle accretion on to a Schwarzschild black hole, 1998a, MNRAS, 298, 835

[14] B. E. Aschenbach, N. Grosso, D. Porquet, P. Predehl, X-ray flares reveal mass and angular momentum of the Galactic Center black hole, 2004, Astron. Astrophys., 417, 71

[15] B. E. Aschenbach, Evidence for GR rotational frame-dragging in the light from the Sgr A* supermassive black hole, 2009, ArXiv.0911.2431

[16] F. Melia, An accreting black hole model for Sagittarius A, 1992, Astrophys. J. Lett., 387, L25

[17] O. Donmez, O. Zanotti, L. Rezzolla, On the development of quasi-periodic oscillations in Bondi-Hoyle accretion flows, 2010, MNRAS, in press, ArXiv.1010.1739 